\title{
Les villes internationales à l'ère de la globalisation et de la décentralisation : quels défis pour le décideur et le gestionnaire? ${ }^{1}$
}

\author{
Caroline Perron, Jacques Gagnon et Panayotis Soldatos \\ Université de Sherbrooke
}

\section{Prolégomènes}

$1^{\circ}$ Depuis les années 1960 - la Province du Québec ayant alors amorcé la version moderne du processus nous entrons dans l'ère des acteurs territoriaux non souverains en relations internationales, dits aussi acteurs sous-nationaux ou, mieux, sous-étatiques, voire infra-étatiques, suivis, dans cette même mouvance, par l'acteur supranational que constituent les Communautés européennes (aujourd'hui l'Union européenne) avec leurs relations extérieures, voire leur politique étrangère et de sécurité commune, sans, certes, oublier, les institutions transnationales.

\begin{abstract}
L'impact sur la configuration internationale fut celui de l'apparition d'un système d'acteurs mixtes, où les acteurs étatiques côtoient, en relations internationales, des acteurs infra étatiques et supranationaux, sans oublier les acteurs transnationaux (firmes transnationales), de plus en plus présents dans les affaires internationales.
\end{abstract}

- identifier la causalité du phénomène;

- définir le profil d'une ville internationale moderne;

- cerner le tissu urbain favorable à un déploiement international réussi;
Les acteurs infra-étatiques, plus spécifiquement (unités fédérées, régions et villes), sous la pression à la fois de réalités internes, poussant à un déploiementaffirmation sur la scène internationale, et de défis internationaux (globalisation), nécessitant une «gestion » sophistiquée de leurs relations internationales, ont su acquérir un profil d'internationalité et se doter d'une planification stratégique de leur déploiement international, le tout sous l'enseigne du concept de paradiplomatie, soit d'une politique internationale, parallèle à celle de l'État-nation (substitutive, surtout en cas de situations internes conflictuelles, ou subsidiaire-d'appoint, en cas de carences de la diplomatie nationale). L'impact sur la configuration internationale fut celui de l'apparition d'un système d'acteurs mixtes, où les acteurs étatiques côtoient, en relations internationales, des acteurs infra étatiques et supranationaux, sans oublier les acteurs transnationaux (firmes transnationales), de plus en plus présents dans les
- formuler un modèle de gouvernance et de planification stratégique gagnant de la paradiplomatie.

\section{Les acquis de l'étude du déploiement international des villes : niveaux et approches d'analyse}

$1^{\circ}$ Sur le plan de la causalité, les facteurs internes et les facteurs externes ont permis de conclure à l'irréversibilité du phénomène ("s'internationaliser ou périr $\gg)^{3}$.

- Au niveau interne, les carences de la diplomatie nationale dans la défense, à l'international, des intérêts des villes, la décentralisation d'appauvrissement, transférant aux villes des rôles publics sans les ressources de leur actualisation, les ambitions identitaires locales de visibilité internationale légitime ont primé dans cette mouvance de paradiplomatie. 
- Sur le plan international, la libéralisation des échanges, les intégrations économiques internationales et supranationales et la transnationalisation des activités économiques, en un mot la globalisation, ont généré, au niveau des villes, leur vulnérabilité économique vu l'élimination progressive des barrières nationales aux échanges, tandis que l'extraordinaire croissance des multilocalisations d'entreprises et de l'IDE (Investissement direct étranger), comme aussi la progression-diffusion mondiale des Nouvelles technologies de l'information et des communications (NTICs), ont ouvert pour les villes la voie (opportunités) à une participation directe et souvent autonome aux relations socio-économiques et culturelles internationales. "Aussi, et plutôt que d'opposer, en cette matière, décentralisation et globalisation, y voyant une relation de contradiction, convient-il d'établir la thèse de la compatibilité (dans une optique d'articulation-agrégation du "domestique" (interne) et de l'international, soit d'“intermestic" ou, autrement, du global et du local, soit de "glocalisme"), l'internationalisation des villes profitant de la décentralisation de l'État-nation, décentralisation qui, à son tour, est renforcée, souvent exacerbée par la globalisation du système économique international qui contribue à la parcellisation de l'espace national, sous la pression des stratégies de multilocalisation des entreprises et de l'investissement direct étranger. »

$2^{\circ}$ Pour ce qui est de la définition, sur une base empirico-comparative, du profil d'internationalité des villes, les démarches accomplies ont permis d'atteindre plusieurs objectifs fondamentaux, soit :

- donner aux décideurs et aux acteurs socio-économiques urbains une feuille de route leur indiquant les traits d'internationalité à acquérir;

- cerner la question de la masse critique des traits d'internationalité et des hiérarchisations afférentes (villes internationales spécialisées, villes internationales généralistes; villes internationales régionales et villes internationales globales; ville-espace et ville-acteur; simples villes internationales et villes internationales directionnelles) ${ }^{4}$.

Depuis quelques années, le profil ainsi économique international des villes modernes est intimement lié à un profil «montant» d'urbanisme culturel à texture culturelle et aux manifestations économiques et d'aménagement du territoire certaines. On y trouve, en effet, l'urbanisme culturel "stricto sensu », la classe créative, l'économie créative ${ }^{5}$.

$3^{\circ}$ Le besoin de cerner le tissu conjonctif urbain, requis pour une internationalisation réussie, est, également, impératif. Il est, en effet, absolument nécessaire de pouvoir fonder l'élan d'internationalisation sur la réalité de tissu conjonctif urbain pour mettre les deux en adéquation féconde et éviter des fuites en avant, soit des déploiements internationaux fragilisés, voire réversibles. "C'est, d'ailleurs dans la grille des composantes de ce "tissu conjonctif urbain" que nous trouvons les facteurs statiques, semi statiques et dynamiques de la compétitivité internationale des villes. ${ }^{6}$

$4^{\circ}$ La complexité d'un système international globalisé et multivarié ("système d'acteurs mixte ») impose la mise sur pied d'un système de gouvernance urbaine innovant et gagnant, articulant dans des schémas de synergies harmonieuses et optimales un faisceau d'espaces, d'institutions, d'acteurs, de politiques, de rôles et de relations.

$5^{\circ}$ Enfin, il importe de procéder à une planification stratégique de la paradiplomatie et du déploiement international avec des institutions modernes et adéquatement calibrées, des alliances stratégiques d'acteurs, internes et externes, des rôles de relais et de leadership urbain dans des réseaux nationaux et internationaux porteurs.

$$
\begin{aligned}
& \text { «C'est, d'ailleurs dans la grille des } \\
& \text { composantes de ce "tissu conjonctif urbain" } \\
& \text { que nous trouvons les facteurs statiques, } \\
& \text { semi statiques et dynamiques de la } \\
& \text { compétitivité internationale des villes. » }
\end{aligned}
$$

S'agissant, plus spécifiquement, des alliances stratégiques de villes (allant bien au-delà des jumelages et s'inspirant, surtout, de la rationalité de celles des entreprises), elles permettent d'atteindre des objectifs stratégiques vitaux, au service d'un déploiement international gagnant, soit : la consolidation-renforcement de liens existants; l'échange d'informations; l'échange-partage de services; le partage d'équipements urbains; la réalisation-promotion commune de travaux de renforcement du tissu conjonctif urbain; l'obten- 
tion, en commun, d'une masse critique de traits d'internationalité ainsi que de fonctions et d'une capacité d'influence internationale; la plus grande capacité d'influence des niveaux supérieurs de gouvernement et d'acteurs; la facilitation d'un processus d'interpénétration du secteur privé des pôles urbains concernés et leur coopération ultérieure; le contrôle organisé des aspects dysfonctionnels d'une concurrence négative entre villes («negative competition »); le renforcement de la notoriété internationale des villes. « Car, si le déploiement international des villes "ne se décrète pas", il se planifie, se "gère" et se projette vers le futur, dans une courbe dynamique et innovante de l'évolution des sociétés internes et du système international. $)^{7}$

La globalisation de l'économie,
combinée aux conflits régionaux et
à la permanence de zones de sous-
développement, a accentué les flux
migratoires internationaux et donné
lieu à l'apparition d'un nouveau
prolétariat international urbain,
en zones d'exclusion.

\section{Les défis sociétaux du déploiement international des villes}

$1^{\circ}$ D'un point de vue d'impact sociétal, l'internationalisation des villes modernes entraîne de sérieux défis pour les populations dont on peut cerner ici les principaux à l'adresse des décideurs, mais aussi de tous ceux qui ont pour mission de réfléchir pour leur solution appropriée (experts, représentants de la société civile).

- Le déploiement international direct des villes pose des problèmes majeurs de coordination des rôles et d'unité dans l'expression de la volonté nationale et des politiques internationales des sociétés.

- L'ouverture aux flux, en général, à l'IDE, en particulier, soumet les villes aux stratégies de parcellisation des entreprises transnationales, ce qui peut conduire à des phénomènes de concassage sociétal (le citoyen se transforme en producteurconsommateur), de déficit démocratique (avec cet éloignement de la vraie décision des élus et son déplacement vers un monde transnationalisé), de coûts sociaux, avec une privatisation des rôles et des orientations en découlant qui pourraient ne pas être suffisamment soucieuses dudit intérêt public.

- Dans cette perforation des sociétés étatiques par les flux internationaux, qui rendent les villes des interlocuteurs directs de l'économie globalisée, d'importantes questions de partage - harmonisation des rôles - entre les secteurs public et privé comme aussi entre les décideurs et la société civile se posent avec acuité. C'est une question à la fois de gouvernance et de politiques sociétales.

$2^{\circ} \mathrm{Si}$ l'on se tourne maintenant vers le devenir et les évolutions de long terme de cette paradiplomatie et, de façon plus générale, de cette mouvance de déploiement international des villes, nous détectons un large éventail de défis d'analyses et d'actions à l'adresse de l'analyste et du décideur-gestionnaire de l'urbain. Sans prétention à l'exhaustivité, nous allons, ciaprès, à l'essentiel.

- La question des finances publiques restera une préoccupation cruciale dans l'optique de ce déploiement international.

En effet, les assainissements budgétaires de nos sociétés modernes, y recherchant des rationalisations dans la globalisation et une attractivité de flux économiques, touchent tous les niveaux de gouvernement, donc celui des villes. Une pénurie de ressources pour le renouvellement d'infrastructures et de services publics urbains vieillissants (équipement urbain : routes, ponts, ports; logements; services de transport, dotation éducationnelle, etc.) en découle et hypothèque l'attractivité en matière de flux internationaux et de localisation d'entreprises étrangères et internationales. Certes, la tentation de trouver dans l'IDE ce manque de ressources internes est grande; son inconvénient, c'est un développement interne soumis aux stratégies de parcellisation des espaces nationaux poursuivies par des acteurs économiques externes en quête de localisations économiquement rentables et pas toujours soucieux de développement interne harmonieux et durable. « La subordination du développement urbain aux stratégies et finalités des acteurs transnationalisés constitue, à cet égard, un processus à éviter. »

- Des contraintes aussi importantes dans le domaine des ressources financières apparaissent, également, dans le contexte de la lutte contre le terroris- 
me : la réorientation des investissements publics vers le secteur de la sécurité provoque une diversion de ressources et prive ainsi les villes d'aides d'État à la hauteur de leurs besoins socio-économiques internes et de déploiement international.

- La privatisation croissante des rôles au sein de la paradiplomatie, et plus généralement de la gouvernance urbaine, comporte des manifestations de sérieux déficits démocratiques: une «paradiplomatie des PDGs », même dans une version mixte (leadership réel privé dans des synergies «publicprivé »), laisse les politiques de déploiement international, tout au moins en partie (partie, toutefois, croissante), aux mains d'acteurs qui n'ont pas la légitimité politique des élus et qui n'organisent pas de façon suffisante et transparente la participation de la société civile à l'élaboration des politiques internationales des villes.

- Des rationalisations structurelles (fusion de villes, privatisation des véhicules de gestion-promotion $\mathrm{du}$ déploiement international des villes, etc.) et fonctionnelles (mégaprojets de développement par exemple, dans l'immobilier commercial et dans les équipements urbains - hiérarchisation des priorités sociétales urbaines en fonction de la grille du déploiement international, etc.), trop calquées sur les rationalités économiques internationales (globalisation, compétitivité, déploiement international), font souvent fi des préoccupations identitaires des citoyens et de leurs collectivités territoriales et locales.

- La globalisation de l'économie, combinée aux conflits régionaux et à la permanence de zones de sous-développement, a accentué les flux migratoires internationaux et donné lieu à l'apparition d'un nouveau prolétariat international urbain, en zones d'exclusion. Aussi, des dislocations sociétales en découlent-elles, limitant, par un déficit afférent de sécurité, de ressources financières disponibles et de qualification de main-d'ouvre, le rythme d'internationalisation des villes du monde occidental développé, en quête de flux économiques, d'attraction d'entreprises, de développement de fonctions internationales.

- La dynamique, fort rafraîchissante, des alliances stratégiques de villes, des années 1980 et 1990, allant alors bien au-delà des fameux jumelages tra- ditionnels, connaît, de nos jours, des difficultés d'épanouissement par la crise de cette paradiplomatie, évoluant au sein d'une gouvernance en transition structurelle (fusions, regroupements transurbains, etc.) et relationnelle (processus de privatisation des rôles toujours à la recherche d'un équilibre de dosage entre le public et le privé, équilibre qui est encore loin d'être atteint et stabilisé).

\section{« D'où le besoin pour un pôle urbain de procéder, de façon rigoureuse, à une radioscopie précise de son profil de ville internationale et de son tissu conjonctif urbain ainsi qu'à une planification stratégique innovante de sa paradiplomatie et de son déploiement international. »}

- Sur le plan des synergies, surtout verticales (entre les acteurs urbains et ceux des niveaux dits supérieurs de gouvernement), des dissonances de politiques et d'actions demeurent et compromettent le déploiement international des villes. Ce dernier est, en effet, tributaire des politiques de ces autres niveaux de gouvernement (politiques d'immigration, monétaires, commerciales, industrielles, énergétiques, de recherche, éducationnelles, etc.). À cet égard, des considérations politiques, voire politiciennes ne permettent pas toujours des arbitrages sociétaux rationnels en faveur de ces nouvelles locomotives du déploiement international dans la Nouvelle économie (tertiarisée, technopolitaine) que sont les pôles urbains. "Aussi, les dirigeants des pôles urbains se débattent-ils, selon les cas de positionnement (faible ou fort), avec des compétences de politiques toujours réduites (par rapport aux niveaux supérieurs de gouvernement), des ressources financières souvent insuffisantes et/ou contrôlées par ces niveaux supérieurs de gouvernement, des assises territoriales héritées du passé et ne correspondant pas toujours aux rationalités spatio-fonctionnelles de l'économie moderne, des "décrochages" sociétaux d'importants segments de la société civile, de ceux qui ne réussissent pas à suivre la logique et la vitesse de ces nouvelles fonctions internationales. »

$3^{\circ}$ Pour conclure, la mouvance " au-delà » (intégration régionale internationale et globalisation) et « en deçà » (décentralisation avec, en l'occurrence, la ma- 
nifestation de déploiement international des villes) de l'État est une tendance lourde de l'évolution sociétale au tournant du $20^{\mathrm{e}}$ siècle et à l'aube du nouveau millénaire, irréversible et contraignante pour nos sociétés, en général, et pour nos pôles urbains, en particulier, déterminant leurs choix et leurs mutations. « D'où le besoin pour un pôle urbain de procéder, de façon rigoureuse, à une radioscopie précise de son profil de ville internationale et de son tissu conjonctif urbain ainsi qu'à une planification stratégique innovante de sa paradiplomatie et de son déploiement international. »

\section{Notes et références}

1 Cette réflexion s'inscrit dans les priorités de déploiement scientifique et de «policy-oriented» de la Chaire J.W. McConnell de développement local (Université de Sherbrooke).

2 Parmi ces nombreuses études, nous pouvons citer ici : Soldatos, P. (1989), Lyon, ville internationale, Lyon, CCIL; Fry, E.H., L.H. Radebaugh et P. Soldatos (1989), The New International Cities Era : The Global activities of North American Municipal Governments, Provo, Brigham Young University; Soldatos, P. (1991), Les nouvelles villes internationales : profil et planification stratégique, Aix-en-Provence, SERDECO; Soldatos, P. (1994), «The "Gateway City" Approach in World Affairs », Revue de droit international de sciences diplomatiques et politiques, $\mathrm{n}^{\circ} 2$; Kresl, P.K. et G. Gappert (dir.) (1995), North American Cities and the Global Economy: Challenges and $\mathrm{Op}$ portunities, Thousand Oaks, SAGE; Soldatos, P. (1996), « La nouvelle génération de villes internationales : phénomène de segmentation des rôles traditionnels de l'Étatnation », dans Chr. Philip et P. Soldatos (dir.), Au-delà et en-deçà de l'État-nation, Bruxelles, Bruylant, p. 203-236; Fry, E.H. (1998), The Expanding Role of State and Local Governments in the U.S. Foreign Affairs, New York, Council on Foreign Relations; Fry, E.H. (2000), The North American West in a Global Economy, Los Angeles, Pacific Council on International Policy; Fry, E.H. et W. McCarlie (2002), Mapping Globalization along the Wasatch Front, Los Angeles, Pacific Council on International Policy; Soldatos, P. (2003), Le rôle international des villes à l'aube du millénaire, Bruxelles, É. Bruylant; Paquin, S.
(2005), Économie politique internationale, Montchrestien/ Clefs, Paris (avec des développements sur la paradiplomatie dans la nouvelle économie politique internationale).

3 Sur cette causalité, voir, notamment: Kresl, P.K. et G. Gappert (dir.) (1995), North American Cities and the Global Economy : Challenges and Opportunities, op. cit.; Fry, E.H. (1998), The Expanding Role of State and Local Governments in the U.S. Foreign Affairs, op.cit.; Soldatos, P. (2003), Le rôle international des villes à l'aube du millénaire, op. cit., p. 27 et suiv.

4 Sur ce débat de définitions, voir notamment : Arrighi De Casanova, E. (1982), Rapport sur les moyens d'accroître le rayonnement international de Paris et de sa région ainsi que des principales métropoles régionales, Paris, avril, p. 5; Polèse, M. (1989), « Montréal International », dans Forum des affaires de Montréal, avril; Labasse, J. (1981), Lyon, ville internationale, juillet, p. 3; Soldatos, P. dans E.H. Fry, L.H. Radebaugh et P. Soldatos, The New International Cities Era: The Global Activities of North American Municipal Governments, op. cit. Il a développé un profil cumulatif de la ville internationale dans son ouvrage $L e$ rôle international des villes à l'aube du millénaire, op.cit., p. 18.

5 Sur ce nouveau profil, voir. : The Creative Economy Initiative: A Blue for Investment in New England's Creative Economy, The New England Council, June 2001; National League of Cities, Tourism and Entertainment as a Local Economic Development Strategy, Washington, DC, 2000; De Brabandere, L. (2002), Le management des idées : de la créativité à l'innovation, Paris, Dunod; De Brabandere, L. et Mokolajczak (2002), Le plaisir des idées : devenir créatif, Paris, Dunod; Peirce, N., Neal Peirce Column, Washington Post Writers Group, July 2, 2000 June 9, 2002 (l'auteur se réfère dans son texte aux réflexions de Alex Krieger (Harvard School of Design) sur les « global cities of amusements » ainsi qu'aux idées de Richard Florida de la Carnegie Mellon University (Pittsburgh), exposées, notamment, dans son récent livre The Rise of the Creative Class).

6 Sur les composantes de ce « tissu conjonctif urbain », voir Soldatos, P. (2003), Le rôle international des villes à l'aube du millénaire, op.cit., p. 63 et suiv. et p. 111.

7 Soldatos, P. (2003), Idem. 


\section{Publicité}

Maîtrise en gestion de projet 